\title{
STRONGLY HOMOGENEOUS SPACES
}

\section{CAROL KITAI}

\begin{abstract}
Spaces satisfying various conditions have previously been called strongly homogeneous spaces and many results about the group of homeomorphisms of such spaces have been proved. However spaces may satisfy some "strongly homogeneous" condition without being homogeneous. In this paper we give a definition of strong homogeneity which implies homogeneity and includes most of the natural examples previously studied.
\end{abstract}

1. Introduction. In recent years several authors have investigated the group of homeomorphisms of certain topological spaces which were called strongly homogeneous spaces. These concepts have been very fruitful (see [1], [2], [3] and [6]), but each author introduced his own variation, some local and some global, to attack his particular problem. Some spaces may even satisfy some "strongly homogeneous" condition without being homogeneous. The object of this paper is to give a definition of strong homogeneity which not only implies ordinary homogeneity and the other strongly homogeneous conditions (or the results deducible from them), but also includes most of the natural examples studied. Moreover this definition can be used as a iocal as well as a global one; for some results, for example the result on ergodic homeomorphisms in $\$ 5$, it suffices that the space contain a strongly homogeneous subset.

If $X$ is any topological space, we denote the group of all self-homeomorphisms of $X$ by $H(X)$. For $U \subset X$ define $U^{\prime}=\{f \in H(X): f(x)=x$ for all $x \notin U\}$. In a metric space $(X, d)$ we denote the $d$-ball of radius $\epsilon$ about $x \in X$ by $N(x, \epsilon)$ and the diameter of a set $U$ by $\delta(U)$.

2. A strong form of homogeneity. We prove that a strongly homogeneous set in a metric space is homogeneous.

Definition 2.1. Let $(X, \tau)$ be a topological space and let $U$ be any open subset of $X, U$ is said to be $B$-freely open if there is a basis $B$ for $\tau$ with the property that if $V, W \in B$ are proper subsets of $U$ with $\bar{W} \cap(\bar{U}-U)=\varnothing$, then for any $v \in V$ there is a homeomorphism $h \in H(X)$ such that

(i) $v \in h(W)$ and $h(\bar{W}) \subsetneq V$,

(ii) $b \in U^{\prime}$.

Definition 2.2. Let $U$ be an open set in a topological space $(X, \tau)$. A

Received by the editors September 6, 1974 .

AMS (MOS) subject classifications (1970). Primary 54C05.

Key words and phrases. Homogeneous, strongly homogeneous.

Copyright $\odot$ 1975. American Mathematical Society 
basis $\mathcal{B}$ for $\tau$ is said to be a free basis for $U$ if every element of $\{B \in \mathcal{B}$ : $B \subset U\}$ is $B$-freely open.

Definition 2.3. Let $U$ be an open set in a topological space $X . U$ is said to be strongly homogeneous (S.H.) if $U$ is $B$-freely open for some free basis $B$ for $U$.

Remark. Every element of a free basis is S.H. The basis of open intervals for the Euclidean topology on the space of real numbers is an example of a free basis.

Lemma 2.4. Let $h$ be a homeomorphism from a space $X$ onto a space $Y$. Then

(i) $U$ is B-freely open in $X$ if and only if $h(U)$ is $h(B)$-freely open in $Y$.

(ii) $B$ is a free basis for $U$ if and only if $h(\Re)$ is a free basis for $h(U)$.

(iii) $U$ is a S.H. set if and only if $h(U)$ is a S.H. set.

Proof. Clear.

Theorem 2.5. If $U$ is a S.H. set in a metric space, then $U$ is homogeneous.

Proof. Let $\mathcal{B}$ be a free basis such that $U$ is $\mathcal{B}$.freely open. Take $a, b$ $\in U$. We show that there is a homeomorphism $F \in H(X)$ such that $F(a)=b$. Let $W_{1}, Y_{1} \in \mathcal{B}$ be proper subsets of $U$ with $a \in W_{1}, b \in Y_{1}, \bar{W}_{1} \cap(\bar{U}-U)$ $=\varnothing, \delta\left(W_{1}\right)<1, \delta\left(Y_{1}\right)<1$. Then there is a homeomorphism $h_{1} \in H(X)$ such that $b \in h_{1}\left(W_{1}\right), h_{1}\left(\bar{W}_{1}\right) \subsetneq Y_{1}, h_{1} \in U^{\prime}$. Let $V_{1}=h_{1}\left(W_{1}\right), a_{1}=h_{1}(a)$, and $B_{1}$ $=h_{1}(\Re)$. By Lemma $2.4, \Re_{1}$ is a free basis. Let $W_{2}, Y_{2} \in \Re_{1}$ be proper subsets of $V_{1}$ with $a_{1} \in W_{2}, b \in Y_{2}, \bar{W}_{2} \cap\left(\bar{V}_{1}-V_{1}\right)=\varnothing, \delta\left(h_{1}^{-1} W_{2}\right)<1 / 2, \delta\left(Y_{2}\right)$ $<1 / 2$ and proceed as above using the fact that $V_{1}$ is $\Re_{1}$-freely open. Continuing in this way we get sequences $\left(W_{n}\right)$ and $\left(V_{n}\right)$ of S.H. sets and homeomorphisms $h_{n} \in H(X)$ such that for all $n=1,2,3, \cdots$ we have

(a) $\bar{V}_{n+1} \subsetneq V_{n}$,

(b) $h_{n}\left(W_{n}\right)=V_{n}$ and $\bar{W}_{n} \cap\left(\bar{V}_{n-1}-V_{n-1}\right)=\varnothing \quad\left(V_{0}=U\right)$,

(c) $\delta\left(h_{1}^{-1} h_{2}^{-1} \cdots h_{n}^{-1} W_{n+1}\right)<1 /(n+1)$ and $\delta\left(W_{1}\right)<1$,

(d) $\delta\left(V_{n}\right)<1 / n$,

(e) $b_{n} \in V_{n-1}^{\prime}\left(V_{0}=U\right)$,

(f) $a_{n}=b_{n} h_{n-1} \cdots h_{1}(a) \in W_{n+1}$.

Let $F_{n}=h_{n} h_{n-1} \cdots h_{1}(n=1,2, \cdots)$. Define the map $F$ by

$$
F(x)=\lim _{n \rightarrow \infty} F_{n}(x) \text { for all } x \in X .
$$

We show that $F(x)$ exists for all $x \in X$, that $F \in H(X)$ and that $F(a)=b$.

Take $x \in X$. If $x \notin U$, then $F(x)=x$. If $F_{n}(x) \notin V_{n}$ for some $n$, then $F(x)=F_{n}(x)$ by (a) and (e). If $F_{n}(x) \in V_{n}$ for all $n$, then by (b) $F_{n-1}(x) \in$ $W_{n}$ for all $n$, so that $d(x, a)<1 / n$ for all $n$ by (c) and (f). Thus $x=a$. 
Also $d\left(F_{n}(a), b\right)=d\left(a_{n}, b\right)<1 / n$ for all $n$ since $W_{n+1} \subset V_{n}$. Therefore $F(a)=b$. Thus $F(x)=\lim _{n \rightarrow \infty} F_{n}(x)$ exists for all $x \in X$. Also by (a), $F$ is one-to-one.

To show $F$ is onto, take $x \in X$. If $x \notin U$, then $F(x)=x$. If $x \in V_{n}$ for all $n$, then by (d), $x=b$, so $F(a)=x$. Otherwise $x \notin V_{n}$ for some $n$. Say $x \notin V_{N}$. Since $F_{N}$ is onto there is an element $y$ of $X$ such that $F_{N}(y)=x$. Thus $F(y)=x$.

We now show that $F$ is continuous. Take $x \in X$. There are two cases to consider. Firstly if $x \neq a$, then there is a positive integer $m$ such that $F_{m}(x) \notin V_{m}$, and so $F(x)=F_{k}(x)$ for all $k \geq m$. Since $\bar{V}_{m+1} \subset V_{m}, F(x)=$ $F_{m+1}^{m}(x) \notin \bar{V}_{m+1}$. Thus there is a $\delta_{1}(x)$ such that $N\left(x, \delta_{1}\right) \subset F_{m+1}^{-1}\left(X-\bar{V}_{m+1}\right)$, so that $d(x, y)<\delta_{1}$ implies $F(y)=F_{m+1}(y)$. Thus $F$ equals $F_{m+1}$ on an open set containing $x$ and so $F$ is continuous at $x$ for $x \neq a$. Now suppose that $x=a$ and let $\epsilon>0$ be given. Let $N$ be a positive integer with $0<$ $1 / N<\epsilon$. Since $F_{N-1}$ is continuous there is a $\delta>0$ such that $N(a, \delta) \subset$ $F_{N-1}^{-1}\left(W_{N}\right)$. So if $d(a, y)<\delta$, then $F(y) \in V_{N}$, and so $d(F(a), F(y))<1 / N<$ $\epsilon$. Thus $F$ is continuous at $x=a$.

It remains to show that $F^{-1}$ is continuous. Take $x \in X$. There are again two cases to consider. If $x \neq b$, then there is an element $z$ of $X$ such that $F(z)=x$ and $z \neq a$. Now $F(z)=F_{N}(z) \notin \bar{V}_{N}$ for some positive integer $N$, so that $F^{-1}(x)=F_{N}^{-1}(x)$. Using the continuity of $F_{N}^{-1}$, a proof similar to the proof of the continuity of $F$ shows that $F^{-1}$ is continuous at $x$. If $x=b$, $F^{-1}(x)=a$. Let $\epsilon>0$ be given and let $N$ be a positive integer with $0<$ $1 / N<\epsilon$. Choose $\delta(b)$ such that $N(b, \delta) \subset V_{N}$. Then $d(b, y)<\delta$ implies $d\left(F^{-1}(y), a\right)<\epsilon$ since $\delta\left(F_{N}^{-1}\left(V_{N}\right)=\delta\left(F_{N-1}^{-1}\left(W_{N}\right)\right)<1 / N\right.$. Thus $F^{-1}$ is continuous.

We have found a homeomorphism $F \in H(X)$ such that $F(a)=b$. Thus $U$ is a homogeneous set.

The following definition is due to Fletcher and McCoy [2].

Definition 2.6. Let $X$ be a perfect, Hausdorff space. $X$ is said to be representable provided that if $F$ is a closed set and $x \notin F$, then $\{h(x): h \epsilon$ $H(X), h=$ identity on $F\}$ contains an open set about $x$.

Remark. Since every S.H. metric space is homogeneous, completely regular and representable, it follows from [2, Theorem 5.2] that every S.H. metric space is a reasonable space, that is, $H(X)$ has a topology such that $H(X)$ is a topological group and the map $\eta: G / C_{x} \rightarrow X$ defined by $\eta\left(g C_{x}\right)=g(x)$ is a home omorphism from $G / C_{x}$ onto $X$ (where $C_{x}=\{h \in H(X): h(x)=x\}$ ).

The Cartesian product of the Cantor set and the one dimensional circle is an example of a space which is homogeneous but not strongly homogeneous. (See Ford [3].)

3. A strongly homogeneous space is a strong local homogene ity. The following definition is due to Ford [3]. 
Definition 3.1. Let $X$ be a Hausdorff space. $X$ is a strong local homogeneity (S.L.H.) if for any neighbourhood of any point $x$, there exists a subneighbourhood $U(x)$ such that for any $z \in U(x)$ there is a homeomorphism $g \in$ $H(X)$ with $g(x)=z$ and $g \in(U(x))^{\prime}$.

Remark. Every S.H. metric space is a S.L.H. It is known that every normed linear space is a S.L.H. [3, Theorem 4.3]. We show that every normed linear space is S.H.

Theorem 3.2. Let $X$ be a normed linear space and let $S=\{x:\|x\|<1\}$. Then $S$ is a S.H. set.

Proof. Define $f: X \rightarrow S$ by

$$
f(x)=x /(1+\|x\|) .
$$

$f$ is a homeomorphism from $X$ onto $S$ with inverse given by

$$
f^{-1}(x)=x /(1-\|x\|)
$$

Let $\mathcal{B}$ be the usual basis for the norm topology on $X$ (so sets $N(x, \epsilon) \in \mathfrak{B}$ ). We show that $S$ is $\mathcal{B}$-freely open. Let $W_{1}, V_{1} \in \mathcal{B}$ be proper open subsets of $S$ with $\bar{W}_{1} \cap(\bar{S}-S)=\varnothing$. Take $v_{1} \in V_{1}$. Now $W_{1} \subset f(W)$ and $V_{1} \supset f(V)$ for some $W, V \in \mathfrak{B}$, and $v_{1}=f(v)$ for some $v \in V$. Say $W=N\left(a, \epsilon_{1}\right)$ for some $a \in X$ and some $\epsilon_{1}>0$. Choose $\epsilon_{2}>0$ such that $\overline{N\left(v, \epsilon_{2}\right)} \subsetneq V$. Define $T: X \rightarrow X$ by

$$
T(x)=\alpha(x-a)+v
$$

where $a=\epsilon_{2} / \epsilon_{1}$. Then $T \in H(X), T(a)=v$ and $T(W)=N\left(\nu, \epsilon_{2}\right)$. Now define $R: X \rightarrow X$ by

$$
\begin{aligned}
R(x) & =\left(f T f^{-1}\right)(x) & & \text { if }\|x\|<1 \\
& =x & & \text { if }\|x\| \geq 1
\end{aligned}
$$

Take $x \in S$. Then

$$
R(x)=\left(f T f^{-1}\right)(x)=\frac{a x+(1-\|x\|)(v-a a)}{1-\|x\|+\|a x+(1-\|x\|)(v-a a)\|} .
$$

So,

$$
\|R(x)-x\|=\left\|\frac{\alpha x+(1-\|x\|)(v-\alpha a)-x(1-\|x\|)-x\|\alpha x+(1-\|x\|)(v-\alpha a)\|}{1-\|x\|+\|\alpha x+(1-\|x\|)(v-\alpha a)\|}\right\| \text {. }
$$

Therefore

$$
\lim _{\|x\| \rightarrow 1^{-}}\|R(x)-x\|=\lim _{\|x\| \rightarrow 1^{-}}\left\|\frac{\alpha x-x\|\alpha x\|}{\|\alpha x\|}\right\|=0
$$

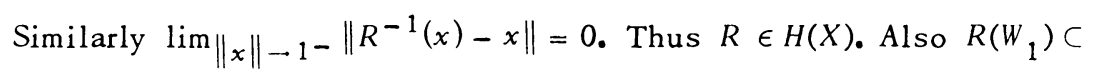


$\left(f T f^{-1}\right)(f(W))=f\left(N\left(\nu, \epsilon_{2}\right)\right)$. So $R\left(\bar{W}_{1}\right) \unrhd V_{1}$. Therefore $S$ is $\mathbb{B}$-freely open. Also, any set of the form $N(x, \epsilon)$ is homeomorphic to $S$ so that $B$ is a free basis for $X$. Thus by Lemma 3.4, $B$ is a free basis for $S$, and so $S$ is a S.H. set.

Corollary 3.3. Every normed linear space is S.H.

We can in fact prove a stronger result for $R^{n}$, namely that if $U$ is any open interval in $R^{n}$, then each pair of proper open subsets $V, W$ of $U$ with $\bar{W} \cap(\bar{U}-U)=\varnothing$ has the property that for each $x \in V$ there is a homeomorphism $h \in H\left(R^{n}\right)$ such that

(i) $x \in h(W)$ and $h(\bar{W}) \subsetneq V$,

(ii) $h \in U^{\prime}$.

4. A strongly homogeneous space is strongly locally setwise homogeneous. The following definitions are due to Brechner [1]. All spaces are taken to be separable metric.

Definition 4.1. Let $X$ be a locally connected continuum and let $G$ be a subgroup of $H(X) . X$ is called locally setwise homogeneous under $G$ if and only if there exist both a basis $B$ of connected open sets of $X$ and a dense subset $A$ of $X$ such that for any $B \in \mathscr{B}$ and $a, b \in A \cap B$ there is a homeomorphism $g \in G$ such that $g \in B^{\prime}$ and $g(a)=b .\{X, A, \mathfrak{B}, G\}$ is called a locally setwise homogeneous structure for $X$. If $X$ is locally setwise homogeneous under $H(X)$, then $X$ is called locally setwise homogeneous, denoted l.s.h.

Definition 4.2. A locally connected continuum $X$ is called strongly locally setwise homogeneous (s.l.s.h.) if and only if there exists a locally setwise homogeneous structure $\{X, A, B, G\}$ for $X$ such that for each $B \in$ $B$ and $x \in A \cap B$ there is a neighbourhood $U$ of $x$ with $\bar{U} \subset B$ satisfying the following property: for each open subset $V$ of $B$ there exists a homeomorphism $h \in G$ such that $h \in B^{\prime}$ and $h(\bar{U}) \subset V$.

We prove that every locally connected, S.H. continuum is s.l.s.h. We use the fact that if $X$ is a connected space and $\mathfrak{D}$ is any open cover of $X$, then any two points $a, b$ of $X$ can be connected by a simple chain of elements of $\mathfrak{T}$, that is, there is a sequence $D_{1}, D_{2}, \ldots, D_{n}$ of elements of $\mathcal{I}$ such that $a \in D_{1}$ only, $b \in D_{n}$ only, and $D_{i} \cap D_{j} \neq \varnothing$ if and only if $|i-j| \leq 1$. It follows that if $\mathcal{B}$ is any basis for the topology of a space $X$ and $U$ is any connected open subset of $X$, then any two points $a, b \in U$ can be connected by a chain $B_{1}, B_{2}, \ldots, B_{n}$ of elements of $\{B \in B: B \subset U\}$.

Theorem 4.3. If $X$ is a locally connected, S.H. continuum, then $X$ is s.l.s.h.

Proof. Let $\mathfrak{I}$ be a free basis for $X$ such that $X$ is $\mathfrak{I}$-freely open. We 
construct a l.s.h. structure $\{X, A, B, G\}$ for $X$ by taking $\mathcal{B}$ to be any basis of connected open sets, $A=X$, and $G=H(X)$. Take $B \in \mathscr{B}$ and $a, b \in B$. Let $D_{1}, D_{2}, \ldots, D_{n}$ be a chain of elements of $\mathfrak{I}$ connecting $a$ and $b$ with $D_{i} \subset B$ for all $i$. Choose $d_{i} \in D_{i} \cap D_{i+1}$ for all $i=1,2, \ldots, n-1$. Then since $D_{1}, D_{2}, \ldots, D_{n}$ are S.H. sets, it follows from Theorem 2.5 that there are homeomorphisms $h_{1}, h_{2}, \ldots, h_{n-1} \in H(X)$ such that

(i) $h_{1}(a)=d_{1}, h_{i+1}\left(d_{i}\right)=d_{i+1}$ for all $i=1, \ldots, n-2, h_{n}\left(d_{n-1}\right)=b$.

(ii) $h_{i} \in D_{i}^{\prime}$ for all $i=1, \ldots, n$.

Let $f=b_{n} b_{n-1} \cdots b_{1}$. Then $f \in H(X), f \in B^{\prime}$, and $f(a)=b$. Thus $X$ is 1.s.h.

Now take $B \in \mathcal{B}$ and $x \in B$. Choose $D \in \mathfrak{T}$ with $x \in D \subset B$ and let $U \in$ $\mathscr{D}$ with $x \in U \subset D, \bar{U} \cap(\bar{D}-D)=\varnothing$. Let $V$ be any open subset of $B$ and choose $v \in V$. Let $\mathscr{T}_{1}$ be the free basis for $X$ consisting of all the elements of $\mathcal{L}$ with the restriction that the basis sets containing $x$ must be connected in $U$. There is a chain $A_{1}, A_{2}, \ldots, A_{n}$ of elements of $\mathscr{L}_{1}$ connecting $x$ and $v$. Since $A_{1} \in \mathscr{T}_{1}, A_{1} \cap U \neq \varnothing$. If there is an integer $m$ such that $A_{m}$ $\cap U \neq \varnothing, A_{m+1} \cap U=\varnothing$, consider the chain $U, A_{m}, A_{m+1}, \ldots, A_{n}$ which connects $x$ and $v$. If $A_{m} \cap U \neq \varnothing$ for all $m=1,2, \ldots, n$, consider the chain $U, A_{n}$. Let $A_{m}^{*}, A_{m+1}^{*}, \ldots, A_{n-1}^{*}$ be elements of $B_{1}$ such that $A_{m}^{*} \subset U \cap$ $A_{m}, A_{m}^{*} \cap\left(\bar{A}_{m}^{m}-A_{m}^{m}\right)=\varnothing$ and $A_{i}^{*} \subset A_{i-1} \cap A_{i}$ with $A_{i}^{*} \cap\left(\bar{A}_{i}-A_{i}\right)^{m}=\varnothing$ for all $i=m+1, m+2, \ldots, n-1$. Then, as in the proof that $X$ is l.s.h., there is a homeomorphism $f \in H(X)$, such that $f \in B^{\prime}$ and $f(\bar{U}) \subset V$. So $X$ is s.l.s.h.

It was proved in [1] that the universal plane curve $M$ is s.l.s.h. However Mazurkiewicz showed that $M$ is not homogeneous (see [4]), and so $M$ is not S.H.

\section{Ergodic homeomorphisms.}

Definition 5.1. Let $X$ be a Hausdorff space. A homeomorphism $T \epsilon$ $H(X)$ is called ergodic if every closed proper subset $A$ of $X$ with $T(A)=A$ is nowhere dense in $X$. The subset of $H(X)$ consisting of the ergodic homeomorphisms is denoted $E(X)$.

Definition 5.2. Let $X$ be a compact metric space. $X$ is called strongly homogeneous at a point $x_{0}$ if there is a basis of neighbourhoods $U_{n}(n=$ $1,2,3, \cdots)$ at $x_{0}$ such that every member of the basis satisfies the following condition:

For every two open subsets $V$ and $W$ of $U_{n}$ with $\bar{W} \cap$

$\left(\bar{U}_{n}-U_{n}\right)=\varnothing$ there is a homeomorphism $h$ from $\bar{U}_{n}$ on-

to itself such that $h(x)=x$ if $x \in \bar{U}_{n}-U_{n}$ and $h(\bar{W}) \subsetneq V$.

Sears proved in [6] that if $X$ is a compact metric perfect space with a strongly homogeneous point, then $E(X)$ is nowhere dense in $H(X)$. A similar proof shows that if $X$ is a compact metric space which contains an open subset $U$ with a free basis, then $E(X)$ is nowhere dense in $H(X)$; in particular if $X$ has a S.H. subset, then $E(X)$ is nowhere dense in $H(X)$. 
Sears also proved in [6] that every point of the Cantor set is a strongly homogeneous point. Similarly, by looking at the Cantor subintervals of rank $r(r=1,2, \cdots)$ (see [5]), we see that the Cantor set is S.H.

The author would like to thank Dr. M. Sears, who suggested this problem to her, for all his helpful advice.

Added in proof. The author has recently improved the result of Theorem 2.5 which now states that if $U$ is a S.H. set in a first countable, regular, $T_{1}$ space, then $U$ is homogeneous. As a result of this, the metric condition needed throughout this paper can be considerably weakened.

\section{BIBLIOGRA PHY}

1. B. L. Brechner, Strongly locally setwise homogeneous continua and their homeomorphism groups, Trans。Amer. Math. Soc. 154 (1971), 279-288. MR 42 $\# 6793$.

2. P. Fletcher and R. A. McCoy, Galois spaces, representable spaces and strongly locally homogeneous spaces, Fund. Math. 73 (1971/72), 85-91. MR 45 $\# 2650$.

3. L. R. Ford, Jr., Homeomorphism groups and coset spaces, Trans. Amer. Math. Soc. 77 (1954), 490-497. MR 16, 609.

4. S. Mazurkiewicz, Sur les contines homogenes, Fund. Math. 5 (1924), 137-146.

5. M. Sears, Topologies on the group of self homeomorphisms of the Cantor set, Mat. Casopis Sloven Akad. Vied. 21 (1971), 227-232. MR 46 \#9299.

6. - On ergodic homeomorphisms, Math. Systems Theory (to appear).

DEPARTMENT OF MATHEMATICS, UNIVERSITY OF THE WITWATERSRAND, JOHANNESBURG, SOUTH AFRICA (Current address)

DEPARTMENT OF MATHEMATICS, MCMASTER UNIVERSITY, HAMILTON, ONTARIO, CANADA 